\title{
Correction to: Efficacy and safety of inhalation budesonide in the treatment of pediatric asthma in the emergency department: a systematic review and meta-analysis
}

\author{
Gollapalle Lakshminarayanashastry Viswanatha ${ }^{1} \cdot$ Hanumanthappa Shylaja ${ }^{2} \cdot$ Krishnadas Nandakumar $^{3}$. \\ Marikunte Venkatanarasappa Venkataranganna ${ }^{4}$ Nunna Bheema Lingeswara Prasad ${ }^{5}$
}

Published online: 19 May 2020

(c) Maj Institute of Pharmacology Polish Academy of Sciences 2020

\section{Correction to: Pharmacological Reports} https://doi.org/10.1007/s43440-020-00098-y

The original version of this article, published on 29 March 2020 unfortunately contains a mistake.

The presentation of the Graphical Abstract and Fig. 1 are incorrect. The correct presentation is given below.

\section{Graphic abstract}

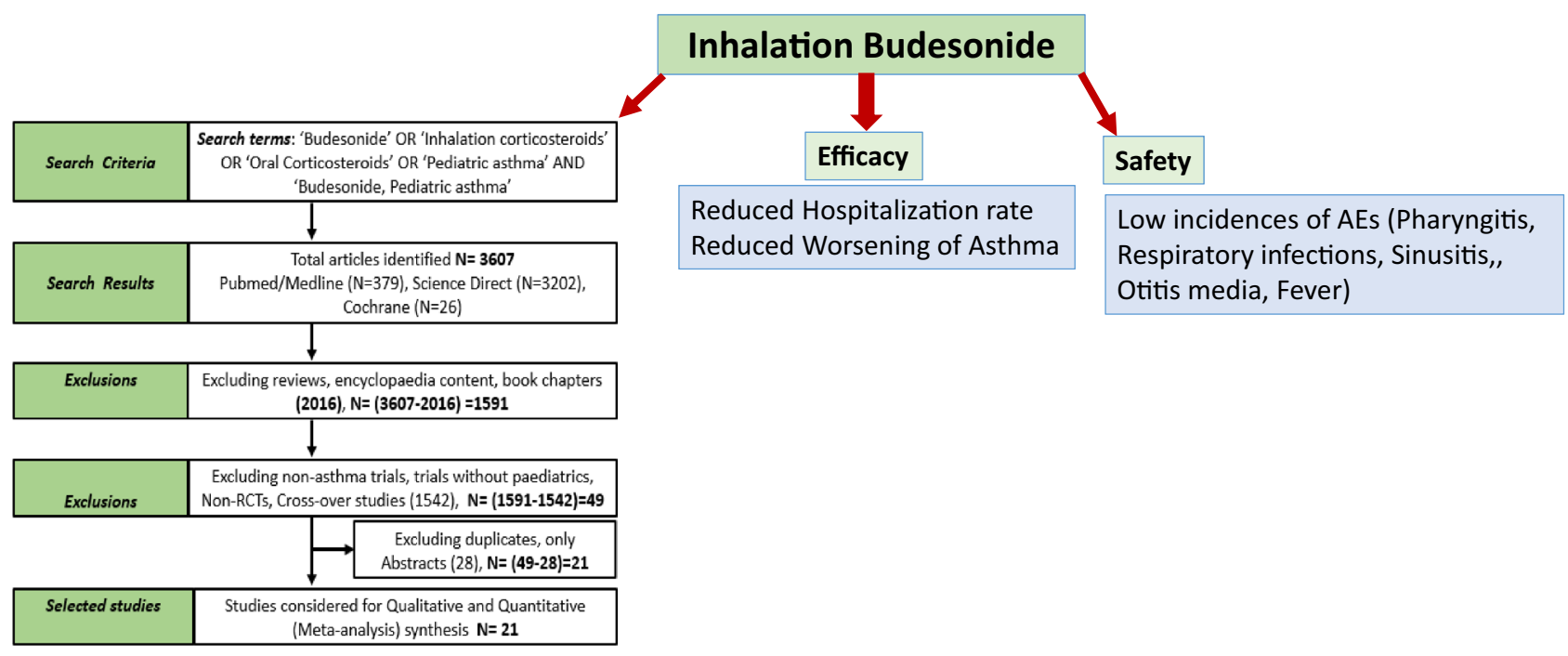

The original article can be found online at https://doi.org/10.1007/ s43440-020-00098-y.

Gollapalle Lakshminarayanashastry Viswanatha glv_000@yahoo.com

1 No.387/511/A, Megalabeedi, Kengeri, Bangalore, Karnataka 560 060, India

2 Kengeri, Bangalore 560060, India
Department of Pharmacology, Manipal College of Pharmaceutical Sciences, Manipal 576104, India

4 Connexios Life Sciences Pvt. Ltd, Bangalore 560060, India

5 OT and PRI, JNT University Anantapur, Ananthapuramu 515002, India 


\begin{tabular}{|c|c|}
\hline Search Criteria & $\begin{array}{l}\text { Search terms: 'Budesonide' OR 'Inhalation corticosteroids' } \\
\text { OR ‘Oral Corticosteroids' OR ‘Pediatric asthma' AND } \\
\text { 'Budesonide, Pediatric asthma' }\end{array}$ \\
\hline Search Results & $\begin{array}{c}\text { Total articles identified } \mathrm{N}=\mathbf{3 6 0 7} \\
\text { Pubmed/Medline (N=379), Science Direct }(\mathrm{N}=3202), \\
\text { Cochrane }(\mathrm{N}=26)\end{array}$ \\
\hline Exclusions & $\begin{array}{l}\text { Excluding reviews, encyclopaedia content, book chapters } \\
\qquad(2016), \mathrm{N}=(3607-2016)=1591\end{array}$ \\
\hline Exclusions & $\begin{array}{l}\text { Excluding non-asthma trials, trials without paediatrics, } \\
\text { Non-RCTs, Cross-over studies (1542), N= (1591-1542)=49 }\end{array}$ \\
\hline & $\begin{array}{c}\text { Excluding duplicates, only } \\
\text { Abstracts (28), } \mathrm{N}=(\mathbf{4 9 - 2 8 )}=\mathbf{2 1}\end{array}$ \\
\hline Selected studies & $\begin{array}{l}\text { Studies considered for Qualitative and Quantitative } \\
\text { (Meta-analysis) synthesis } \mathrm{N}=\mathbf{2 1}\end{array}$ \\
\hline
\end{tabular}

Fig. 1 PRISMA flow chart 